\title{
The Wound Healing Effect of Hydnocarpi Semen Extract on Ulcer in Diabetic Mice
}

\author{
Geum Seon LeE ${ }^{1,2}$, Ji Young $\mathrm{CHOl}^{1,2}$, Yun Jung $\mathrm{CHOl}^{1,2}$, Dong Sool $\mathrm{YIM}^{1}$, Tae Jin KANG ${ }^{1,3, *}$, and Jae Hoon $\mathrm{CHEONG}^{1,2, *}$ \\ ${ }^{1}$ College of Pharmacy, ${ }^{2}$ Uimyung Research Institute for Neuroscience, \\ ${ }^{3}$ Institute of Chronic Disease, Sahmyook University, Seoul 139-742, Republic of Korea
}

(Received June 8, 2010; Revised July 7, 2010; Accepted July 16, 2010)

\begin{abstract}
The extract from Hydnocarpi Semen (HS) has been used to treat leprosy and its anti-inflammatory activity has been reported. However, the effect of HS on the treatment of diabetic or peripheral ulcer is not well known. We therefore examined its wound healing effects on ulcer area in diabetic mice. GC and GC/MS analysis with the total extract of HS show that the main constituents of the extract are chaulmoogric acid, hydnocarpic acid, and gorlic acid. Whereas HS showed wound healing effect in diabetic ulcer, there was no hypoglycemic effect in diabetic mice. The treatment of HS extract significantly decreased the level of total WBC and neutrophils in mice compared to control mice. Cutting ulcer was induced by the round-shaped punch on the backside of diabetic mice and the extract of HS was given orally or topically. The wound area score significantly decreased after treatment of HS at dose of $50 \mathrm{mg} / \mathrm{kg}$. The treatment of HS also induced the activation of macrophages and increased the production of IL-12 and TNF- $\alpha$ in macrophages, indicating that the wound healing by HS extract is associated with the inflammatory effect via the activation of macrophages. Our results suggest that HS extract can be a new therapeutic candidate for treatment of diabetic ulcer.
\end{abstract}

Keywords: Diabetic ulcer, Hydnocarpi Semen, Wound healing, Cytokine, Macrophages

\section{INTRODUCTION}

An ulcer is a local defect or excavation of the surface of an organ or tissue and is produced by the sloughing of inflammatory necrotic tissue. Ulceration can occur only when an inflammatory necrotic area exists on or near the surface. It is most commonly encountered in inflammatory necrosis of the mucosa of the mouth, stomach, intestines, or genitourinary tract and subcutaneous inflammations of the lower extremities in older persons who have circulatory disturbances that predispose to extensive necrosis. During the acute stage, there is intense polymorphonuclear (PMN) infiltration and vascular dilation in the margins of the defect.

Diabetes mellitus (DM) is a chronic hyperglycemic disorder, considered as a syndrome rather than a simple

${ }^{*}$ Corresponding authors

Tel: +82-2-3399-1605 (Cheong JH), +82-2-3399-1617 (Kang TJ)

Fax: +82-2-3399-1608 (Cheong JH), +82-2-3399-1617 (Kang TJ)

E-mail: cheongjh@syu.ac.kr (Cheong JH), kangtj@syu.ac.kr (Kang TJ) disease. Complicated metabolism and increased incidence of infections are its clinical hallmarks, and several mechanisms seem to be involved, such as neuropathy, small vessels and metabolic alterations, which are mostly associated with the chronicity of the disease. However, there is little information about the early pathological processes in diabetes.

For the study of anti-diabetic agents, STZ (N-[methylnitrocarbamoyl]-D-glucosamine) induced hyperglycemia in rodents is considered to be a good preliminary screening model (Ivora et al., 1989) and is widely used since STZ is known to destroy pancreatic $\beta$-cells (Lukic et al., 1998; Spinas, 1999). STZ is a potent methylating agent for DNA and acts as nitric oxide donor in pancreatic cells. $\beta$-cells are particularly sensitive to damage by nitric oxide and free radical scavenging enzymes (Lukic et al., 1998; Spinas, 1999; Meghana et al., 2007).

Hydnocarpi Semen (HS) has been used as a traditional medicine for treating subjects with leprosy and its antimicrobial, antioxidant, anti-fungal, and anti-inflammatory activities were reported (Levy, 1975; Sharma and Hall, 
1991; Oommen et al., 1999; Oommen, 2000). However, the effect of HS on the treatment of diabetic or peripheral ulcer is not well known. In our natural products screening, HS has been shown to have anti-ulcer activity. In this study, we investigated HS anti-ulcer activity in streptozotocin-induced diabetic mice.

\section{MATERIALS AND METHODS}

\section{Reagents}

Streptozotocin (STZ) was obtained from Sigma Aldrich Korea (Yongin, Kyunggi-do, Korea). Thiopental sodium was from Choongwae Pharma Corp. (Anyang, Kyunggi-Do, Korea). Hydnocarpi Semen (HS) seeds were purchased from Kyoungdong Oriental drug store (Seoul, Korea). ELISA kits (DuoSet) for IL-12 and TNF- $\alpha$ were obtained from R\&D system (Woongbe MeDiTech, Inc. Seongnam, Kyunggi-Do, Korea).

\section{Animals}

The male ICR mice (20-25 g) used in this study were obtained from Hanlim Laboratory Animals Co. (Hwasung, Kyunggi-Do, Korea). All animals were maintained on a standard light-dark cycle, at ambient temperature $(23 \pm$ $2^{\circ} \mathrm{C}$ ) and humidity ( $55 \pm 10 \%$ ) with free access to chow pellets and water. All animals were acclimated to their home cages for at least 7 days before testing. The experimental groups, consisting of 8-10 animals per drug and dose, were chosen by means of a randomized schedule. Animal treatment and maintenance were carried out in accordance with the Principle of Laboratory Animal Care $(\mathrm{NIH}$ publication No. 85-23 revised 1985) and the Animal Care and Use Guidelines of Sahmyook University, Korea.

\section{Plant material and extraction}

Dried HS seeds were extracted with methanol. The crude seeds were crushed and extracted three times in simmering $80 \%(\mathrm{v} / \mathrm{v})$ aqueous methanol $(2,500 \mathrm{ml}$ each time). The extracts were combined and the solvent was removed by rotary evaporator (Büchi, Swiss). The raw extracts were pooled, filtered, and evaporated to dryness in a rotary vacuum evaporator.

\section{Induction of diabetes by streptozotocin}

STZ is widely used as a hyperglycemic inducer in rodents (Ivora et al., 1989) since STZ is known to destroy pancreatic $\beta$-cells (Lukic et al., 1998; Spinas, 1999). Therefore, diabetes was induced by injection of $60 \mathrm{mg} / \mathrm{kg}$ of STZ intravenously (i.v.) via tail vein. One week after STZ administration, the hyperglycemic mice (glucose levels being
$200 \mathrm{mg} / \mathrm{dl}$ ) were selected and used for ulcer induction. Non-treated mice were used as control group.

\section{Measurement of glucose level in blood}

Blood was obtained from tail vein and blood glucose levels were measured using MediSense ${ }^{\circledR}$ Optium ${ }^{\mathrm{TM}}$ Blood Glucose Electrodes (Abbott Laboratories, Alameda, CA USA) at indicated times (days) in the presence or absence of HS treatment.

\section{Ulcer induction and treatment of HS for wound healing}

Mice were shaved with animal clippers to expose the entire dorsal surface. Deep anesthesia was induced with thiopental sodium $(50 \mathrm{mg} / \mathrm{kg}$, i.p.). The cutting ulcer was induced by the round-shaped $0.7 \times 0.7 \mathrm{~cm}^{2}$ stainless steel punch on the backside of mice. After the induction of cutting injury, mice were treated orally or by dermal application, respectively, with the extract from HS. The surface area of the wound was measured by tracing the boundary of open wound on semi-transparent paper and calculation of area was done by using a graph paper.

\section{Quantification of white blood cells (WBCs)}

Mice were sacrificed on 1, 3, 7, 10 and 14 days after treatment of HS and cardiac blood was obtained by thoracotomy from mice and used for complete blood count (CBC). The number of WBCs and neutrophils were determined by counting using an automatic hematology analyzer, Hemavet 850 (CDC tech, Oxford, CT, USA).

\section{Preparation of murine peritoneal macrophages and culture}

Primary peritoneal macrophages were obtained from Crl: CD-1 (ICR) BR mice (Jackson Labaratories, Bar Harbor, ME) 4 days after intraperitoneal (i.p) inoculation of $3 \mathrm{ml}$ of $3 \%$ thioglycollate. Peritoneal fluid was drawn through the abdominal wall with a 23-gauge needle. Fluid from mice was pooled, washed, total cell counts determined using a hemacytometer, and the remaining fluid was centrifuged at $380 \times \mathrm{g}$ for $10 \mathrm{~min}$ at $4^{\circ} \mathrm{C}$ (Kang et al., 2005). Washed cell suspensions were adjusted to $10^{6}$ macrophages per $\mathrm{ml}$ in culture medium containing RPMl1640 with fetal bovine serum (FBS) $10 \%$ and $50 \mu \mathrm{g} / \mathrm{ml}$ gentamicin, and incubated in 24-well cell culture plate in $5 \% \mathrm{CO}_{2}$ at $37^{\circ} \mathrm{C}$ overnight before exposure to $\mathrm{HS}$.

\section{Measurement of cytokines}

Murine macrophages were treated with the HS at a dose-dependent manner. In some experiment, macrophages were pre-treated with LPS (O111:B4; Sigma) for activation 
of cells. Culture supernatants were assayed for mouse IL-12, and TNF- $\alpha$ by ELISA according to the manufacturer's instruction.

\section{Analysis of fatty acids by GC and GC mass}

The total extract of HS (25 mg) were collected, added with $1.5 \mathrm{ml}$ of $2 \mathrm{M} \mathrm{NaOH}$ in methanol and after vortex, were kept in an oven with a temperature of $85^{\circ} \mathrm{C}$ for $6 \mathrm{~min}$. After cooling, $2 \mathrm{ml}$ of $12 \%$ BF3 in methanol was added in the total extract of $\mathrm{HS}$ and again kept in an oven under a temperature of $100^{\circ} \mathrm{C}$, heated for $15 \mathrm{~min}$. The fatty acid methyl esters (FAMEs) were then extracted once with $1 \mathrm{ml}$ of $\mathrm{n}$-hexane $(95.0 \%)$ and identified by GC/MS and quantified by GC. Mass detector operating in $70 \mathrm{~V}$ electron ionization (EI) mode. One hundred microliter of sample was injected into the GC/MS system with split-less mode.

Carrier gas $(\mathrm{He})$ flow was set at $0.8 \mathrm{ml} / \mathrm{min}$. The temperature of the injection port was set at $230^{\circ} \mathrm{C}$ and oven temperature was programmed to initiate at $120^{\circ} \mathrm{C}$ for $1 \mathrm{~min}$, the temperature was raised to $200^{\circ} \mathrm{C}$ at a rate of $20^{\circ} \mathrm{C} / \mathrm{min}$ and held for $4 \mathrm{~min}$, increased to $230^{\circ} \mathrm{C}$ at a rate of $5^{\circ} \mathrm{C} / \mathrm{min}$ and held there for $10 \mathrm{~min}$. The injection volume was $100 \mu \mathrm{l}$ in the split-less injection mode. A capillary column (HPFFAP capillary, $30 \mathrm{~m} \times 0.32 \mathrm{~mm}$ I.D., $0.25 \mu \mathrm{m}$ film thickness; Agilent Technologies Inc, Santa Clara, CA, USA) was employed. The total chromatographic run time was 30 min; however, a subset between 330 and 1,000 s was used for the analysis of FAMEs. Distribution type of fatty acids methyl esters was investigated, $\mathrm{m} / \mathrm{z}=74$ ion chromatogram. Quantization of methyl esters of fatty acids was performed by integration of appropriate peak areas in Total Ion Chromatogram (TIC).

\section{Statistical analysis}

All data were expressed as the mean \pm S.E.M. For statistical evaluation, one-way ANOVA was used. When significant differences were found, Newman Keuls test was used as a post-hoc test. Significance was set at $p<0.05$.

\section{RESULTS}

\section{The induction of diabetic mice by STZ and blood-glucose level}

As shown in Fig. 1, treatment of mice with STZ, an inducer of diabetes, increased their blood glucose levels comparably higher than non-treated mice. The blood glucose levels of diabetic animals with blood glucose levels of above $200 \mathrm{mg} / \mathrm{dl}$ no longer increased 17, 24, and 31 days after treatment of the HS extract mean while treatment of STZ increased the blood-glucose level in mice in the ab-

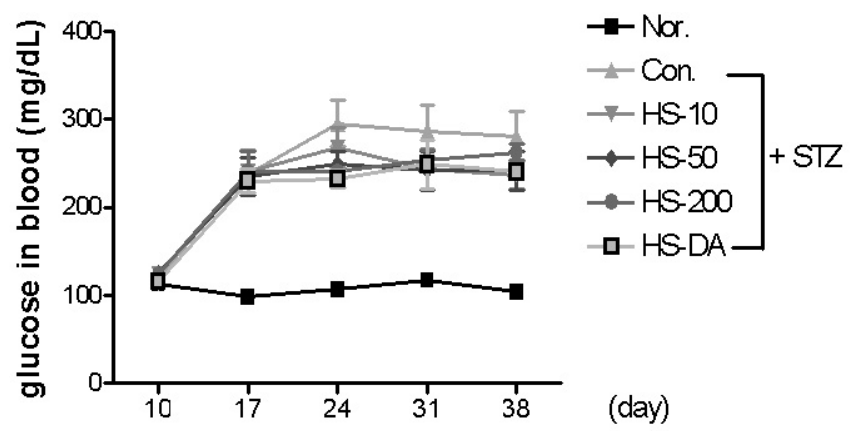

Fig. 1. The effect of HS on blood-glucose level in diabetic mice (10 mice per each group). Diabetic mice were induced by injection of STZ (60 mg/kg mice) intravenously and bloodglucose levels were measured at indicated times in the absence or presence of HS treatment.

sence of HS treatment (Fig. 1). However, treatment of diabetic mice with $\mathrm{HS}$ extract did not reduce the blood glucose concentrations even at high dose of the HS extract indicating that there is no hypoglycemic effect of HS extract in the diabetic animal.

\section{Anti-ulcer effect of HS in diabetic mice}

Diabetic mice were induced by injection of STZ intravenously (i.v.) as described in Materials and Methods. One week after STZ administration, the hyperglycemic animals were selected and used for anti-ulcer effect of HS extract. The surface area of wound healing was measured to demonstrate the anti-ulcer effect of HS on skin ulcer induced by cutting injury in diabetic animals. Treatment of animals with HS by oral and dermal administration, respectively, increased anti-ulcer effect in skin ulcer lesion. The wound area score was significantly decreased in a dose dependent manner and there is no lesion at 2 weeks after oral administration of HS compared to non-treated control. Dermal application with the extract shows a similar result with oral administration (Fig. 2). Especially, this reduced wound area was shown significantly at doses of $50 \mathrm{mg} / \mathrm{kg}$ compared to non-treated control (Fig. 2B, C) suggesting that HS extract can be used to treat diabetic ulcer by healing ulcer lesions.

\section{WBC and neutrophil levels in mice}

During the acute stage of DM, there is intense polymorphonuclear (PMN) infiltration. We therefore measured the level of white blood cell (WBC) such as neutrophils in peripheral blood from diabetic mice treated with HS extract. Cardiac blood was obtained from mice 14 days after treatment of $\mathrm{HS}$ and $\mathrm{CBC}$ was performed. Oral administration of HS (50 and $200 \mathrm{mg} / \mathrm{kg}$ ) significantly decreased the levels of total WBC and neutrophils compared to none- 
A

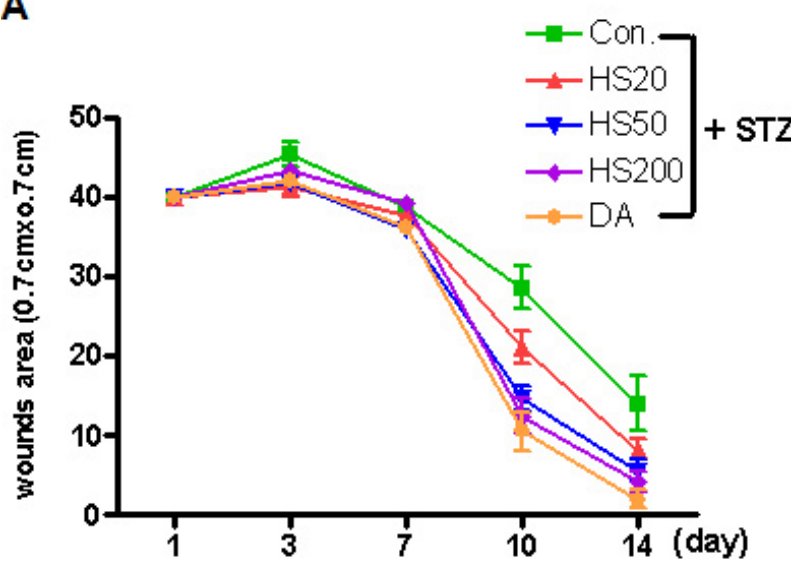

B

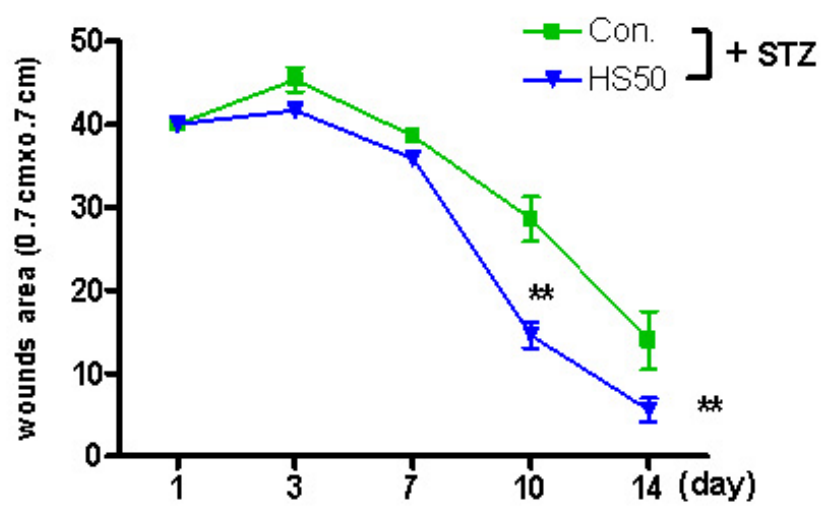

C

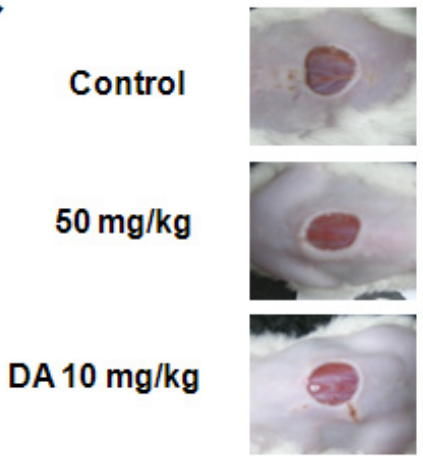

1

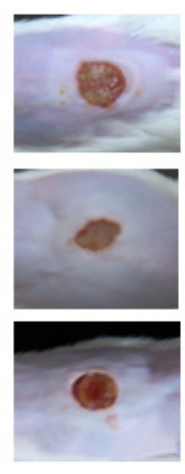

3

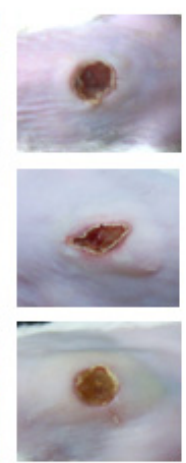

7

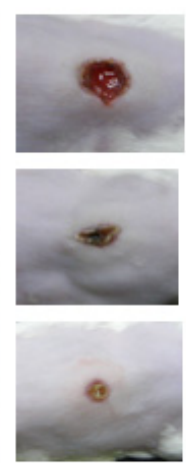

10

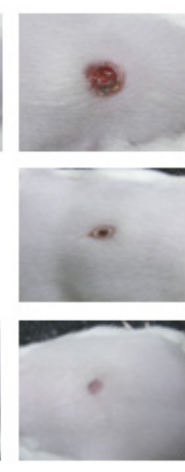

14 Day

Fig. 2. Anti-ulcer effect of HS on the skin ulcer. The skin ulcer in diabetic mice was induced by cutting with stainless steel punch on the backside of mice. (A, B) The surface area of wound healing was measured after oral administration of HS at a dose dependent manner $(20,50$, and $200 \mathrm{mg} / \mathrm{kg}$ ) or dermal application (DA, $10 \mathrm{mg} / \mathrm{kg}$ of HS) for indicated times. Each data are expressed as mean \pm SEM of injured area score for 14 days. ${ }^{* *} p<0.01$ versus control. DA means dermal application of HS. (C) The surface area of wound healing was measured after oral treatment of HS at dose of $50 \mathrm{mg} / \mathrm{kg}$ or dermal application (DA, $10 \mathrm{mg} / \mathrm{kg}$ of HS). Non-treated mice were used as a control.

treated diabetic mice (Fig. 3).

\section{The effect of HS extract on cytokine production in macrophages}

Wound healing is a complex but orderly phenomenon involving a number of processes. The mechanisms underlying most of processes involve the mediators of acute inflammation. Since HS promotes wound healing in vivo, we asked whether the anti-ulcer effect of HS is due to the activation of macrophages to produce inflammatory cytokines. Macrophages were treated with HS extract at a dose-dependent manner and the level of several cytokines, such as TNF- $\alpha$, and IL-12 was measured with the cell culture supernatant. The treatment of HS extract induced the activation of macrophages and increased the production of IL-12 and TNF- $\alpha$ in macrophages at dose de- pendent manner (Fig. 4). These results show that wound healing activity is, partially, due to the activation of macrophages and cytokine secretion.

\section{Analysis of fatty acid in total extract of HS by GC/MS}

We analyzed the components of total extract from $\mathrm{HS}$ by GC/MS and GC analysis. As we expected, the major constituent of fatty acid in total extract of HS consists of chaulmoogric acid $(6.69, \mathrm{C} 18)$, hydnocarpic acid $(9.577, \mathrm{C} 16)$ and gorlic acid (12.97, C18) (Fig. 5A). GC-MS Analysis with the extract of HS shows the similar results and the totoal extract also include chaulmoogric acid (17:13.5, C18), hydnocarpic acid (31.13.7, C16) and gorlic acid (37.17.7, C18) (Fig. 5B). 

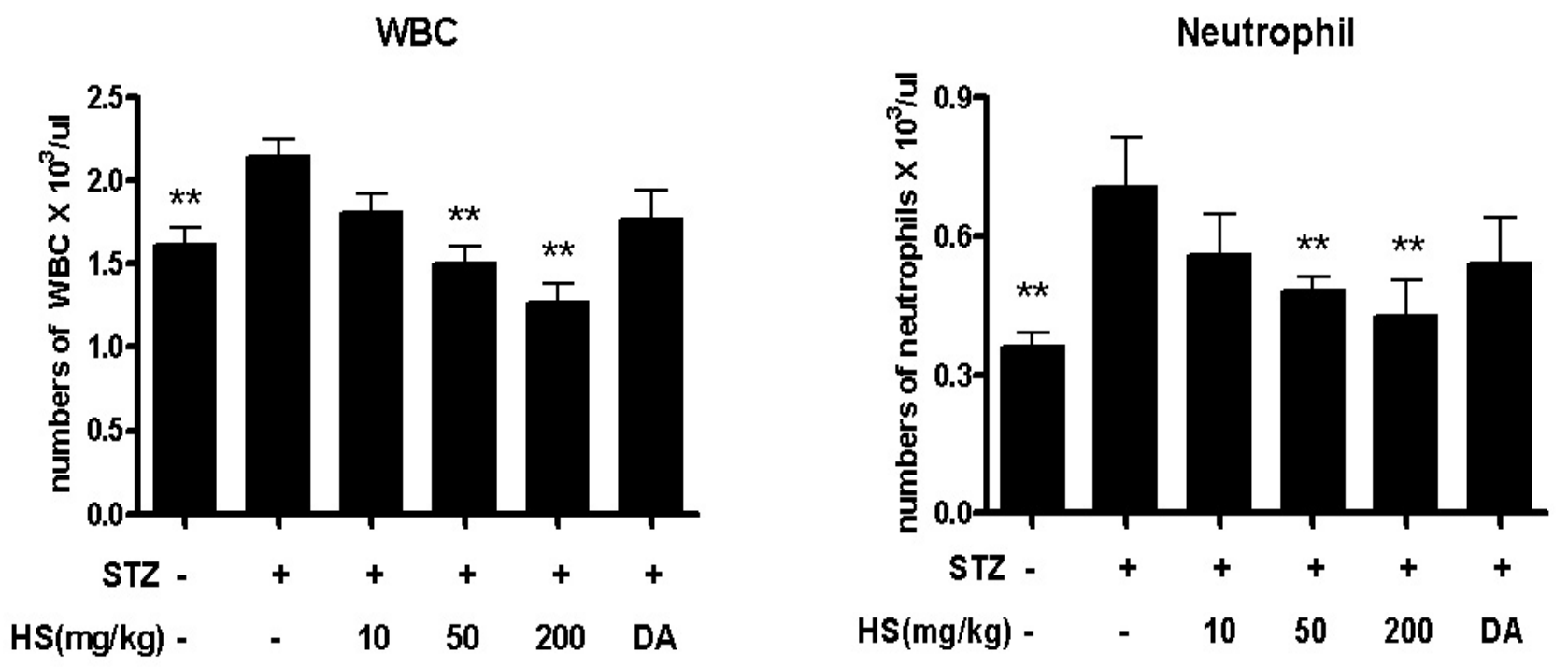

Fig. 3. WBC and neutrophil levels in diabetic mice treated with $\mathrm{HS}(\mathrm{n}=10)$. Cardiac blood was obtained by thoracotomy of diabetic mice at 14 days after treatment of HS and used for Complete Blood Count (CBC). Data were represents the mean \pm SEM of WBC and neutrophil levels in blood. ${ }^{* *} p<0.01$ compared to control mice treated with only STZ.

A

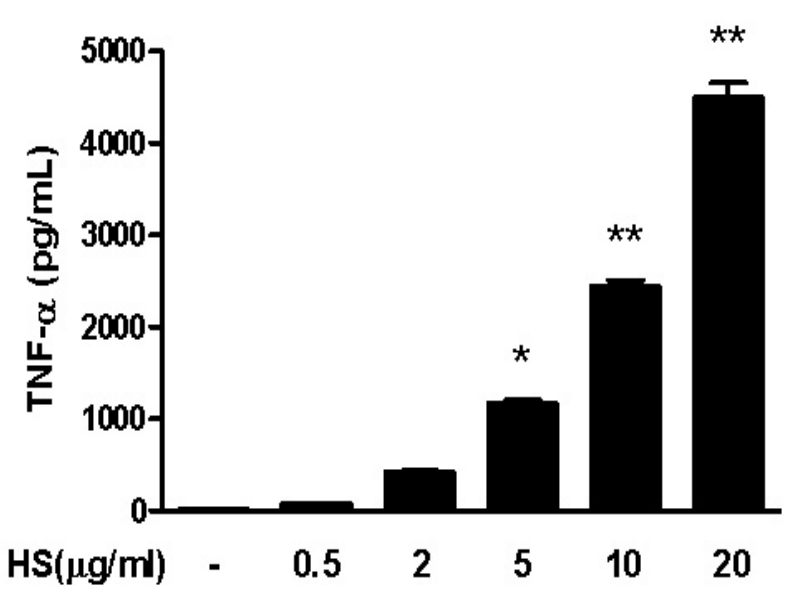

B

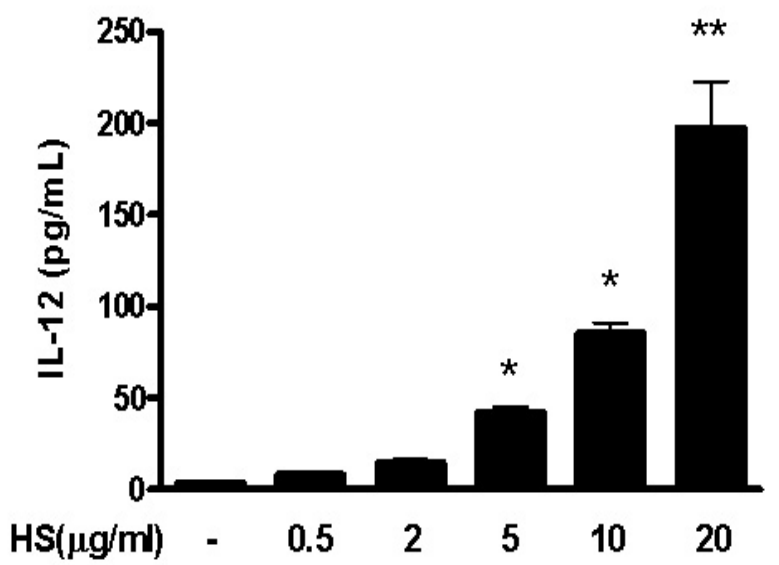

Fig. 4. The effect of HS extract on the production of inflammatory cytokines by macrophages. Macrophages from ICR mice were treated with the extract of HS at a dose dependent manner for $24 \mathrm{~h}$, and cell culture supernatants were collected for IL-12 and TNF- $\alpha$ assay. Data are representative of at least two independent experiments, each done in triplicate; ${ }^{*} p<0.05$, ${ }^{* *} p<0.01$ compared to non-treated cells.

\section{DISCUSSION}

The aim of this study is to evaluate the anti-ulcer activity by HS extract on the ulcer area in diabetic mice. Among the long-term complications of DM, foot or peripheral ulcer is the most serious problem. Most ulcers have a mixed etiology that is neuropathy, peripheral ischemia and immune disorder. Infection is usually the consequence rather than the cause of foot ulcers and can cause substantial delay in healing. DM is also a condition which is associated with a variety of connective tissue abnormalities. The collagen content of the skin is decreased as a result of reduced biosynthesis and/or accelerated degradation of newly synthesized collagen. These qualitative and quantitative abnormalities contribute to the impaired wound healing observed in diabetes (Goodson and Hunt, 1979). 

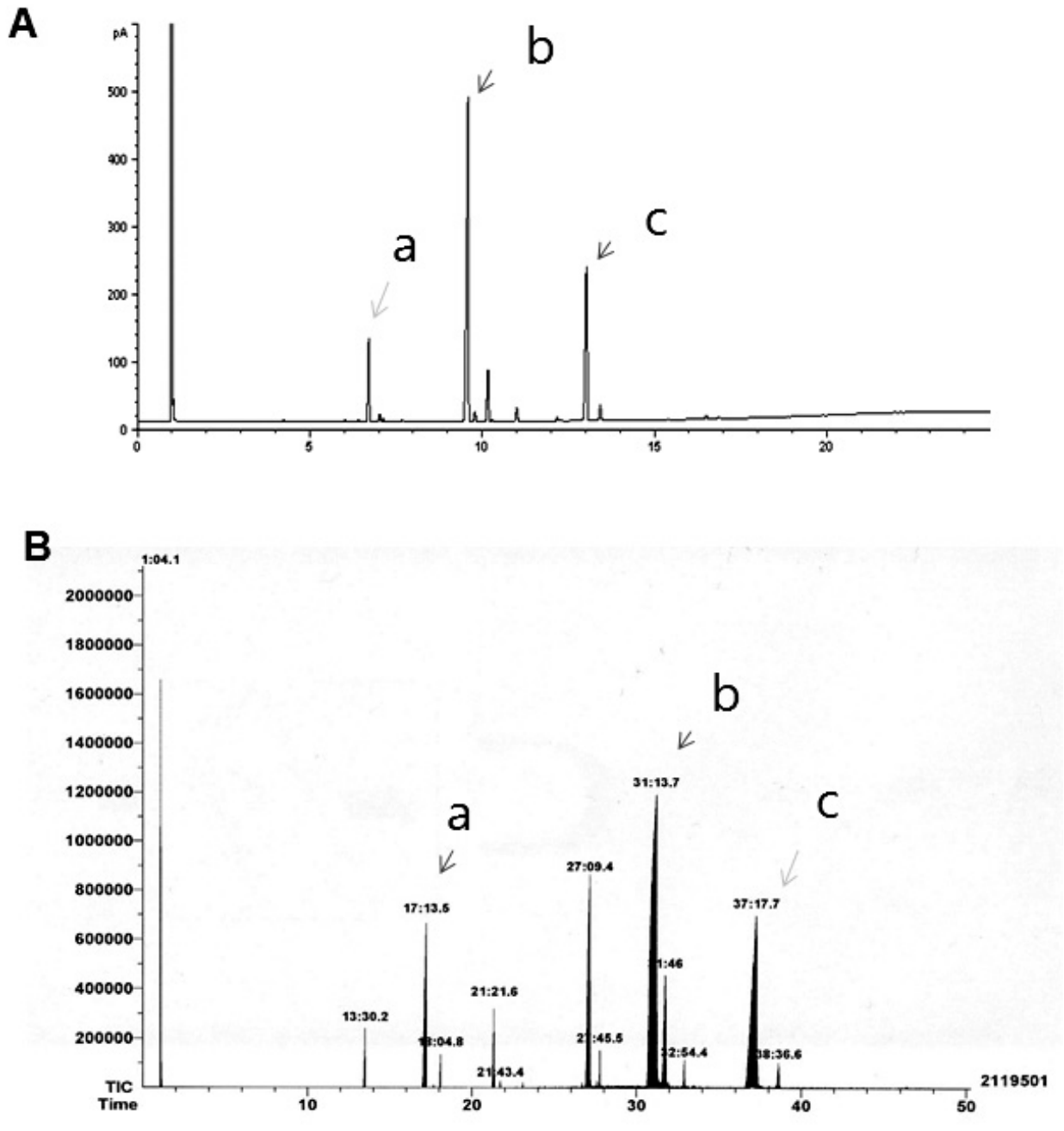

Fig. 5. Typical chromatogram of fatty acids from total extract of Hydnocarpi Semen by (A) GC and (B) GC/MS Analyzer (HPFFAP Column). Each peak indicates (a) Chaulmoogric acid, (b) Hydnocarpic acid, (c) Gorlic acid.

Despite of many efforts to develop hypoglycemic or anti-diabetic ulcer agents, there is still a large demand for developing new agents (Ajabnoor, 1990; Chithra et al., 1998; Park et al., 2009). During our research for novel anti-diabetic ulcer agents from natural products, we found that the HS extract has wound healing activity in mice. However, although the HS extract has the anti-ulcer effect in diabetic mice, it failed to reduce the blood glucose level in diabetic (Fig. 1, 2).

Therefore this study focused on the wound healing in the dermal tissue following cutting injury in diabetic mice induced by STZ. Wound healing, a fundamental response to tissue injury, is a complex process involving a highly regulated series of biological events and occurs by a process of connective tissue repair. The wound area score was significantly decreased at a dose dependent manner by treatment of HS extract (Fig. 2). Our result suggests that the reduction of WBC and neutrophil in the mice treated with HS (Fig. 3), through an unknown mechanism, is asso- ciated with anti-ulcer effect. It is possible that the enhanced healing of wounds in diabetic mice by HS is a result of its inflammatory activity and its capacity to stimulate wound healing. However, pharmacological investigations are necessary to confirm its mechanism of action and its anti-diabetic potential.

In the absence of inflammation, wounds would never heal. The present study demonstrated that the HS extract accelerates wound healing in diabetic animal via the activation of macrophages showing increase of inflammatory cytokines, such as TNF- $\alpha$ and IL-12, at dose dependent manner (Fig. 4). The important relationship of wound healing has been found to exist between fibroblast, keratinocytes, and resident dermal cell (Raghow, 1994). TNF- $\alpha$ has an important role in the activation of vascular endothelial cell, the induction of angiogenesis, and proliferation of fibroblast. During our research for novel anti-ulcer agents from natural products, we found that the HS extract increased the production of pro-inflammatory cytokine, TNF- $\alpha$ 
in a dose-dependent manner (Fig. 4A). Interestingly, the $\mathrm{HS}$ did not increase the TNF- $\alpha$ production of macrophages activated with LPS, suggesting that the HS may protect the cell and tissue from injury or destruction at high concentration of TNF- $\alpha$ (Data not shown).

For now, the mechanism on the anti-ulcer activity by the HS is unknown. Therefore, we will study the role of HS extract on normal function of the connective tissue and angiogenesis. In addition, our future study will be to probe each of the chemical constituents of HS extract showing the wound healing effect and thereafter establish the mechanism of their wound healing activity.

\section{ACKNOWLEDGMENTS}

This work was supported by the Sahmyook University Research Fund in 2009.

\section{REFERENCES}

Ajabnoor, M. A. (1990). Effect of aloes on blood glucose levels in normal and alloxan diabetic mice. J. Ethnopharmacol. 28, 215-220.

Chithra, P., Sajithlal, G. B., and Chandrakasan. G. (1998). Influnce of aloe vera on the healing of dermal wounds in diabetic rats. J. Ethnopharmacol. 59, 195-201.

Goodson, W. H. 3rd. and Hunt, T. K. (1979). Wound healing and the diabetic patient. Surg. Gynecol. Obstet. 149, 600-608.

Ivora, M. D., Paya, M. and Villar, A. (1989). A review of natural products and plants as potential antidiabetic drugs. $J$.
Ethnopharmacol. 27, 243-275.

Kang , T. J., Fenton, M. J., Weiner, M. A., Hibbs, S., Baillie, L. and Cross, A. S. (2005). Murine macrophages kill the vegetative form of Bacillus anthracis. Infect. Immun. 73, 7495-7501.

Levy, L. (1975). The activity of chaulmoogra acids against Mycobacterium leprae. Am. Rev. Respir. Dis. 111, 703-705.

Lukic, M. L., Stosic-Grujicic, S. and Shahin, A. (1998). Effector mechanisms in low dose sterptozotocin induced diabetes. Dev. Immunol. 6, 119-128.

Meghana, K., Sanjeev, G. and Ramesh, B. (2007). Curcumin prevents streptozotocin-induced islet damage by scavenging free radicals: a prophylactic and protective role. Eur. J. Pharmacol. 577, 183-191.

Oommen, S. T. (2000). The effect of oil of hydnocarpus on excision wounds. Int. J. Lepr. Other. Mycobact. Dis. 68, 6970.

Oommen, S. T., Rao, M. and Raju, C. V. (1999). Effect of oil of hydnocarpus on wound healing. Int. J. Lepr. Other. Mycobact. Dis. 67,154-158.

Park, J. S., Yang, J. S., Hwang, B. Y., Yoo, B. Y. and Han, K. (2009). Hypoglycemic effect of Yacon tuber extract and its constituent chlorogenic acid, in streptozotocin-induced diabetic rats. Biomol. \& Ther. 17, 256-262.

Raghow, R. (1994). The role of extracellular matrix in post inflammatory wound healing and fibrosis. FASEB. J. 8, 823831.

Sharma, D. K. and Hall, I. H. (1991). Hypolipidemic, anti-inflammatory, and antineoplastic activity and cytotoxicity of flavonolignans isolated from Hydnocarpus wightiana seeds. $J$. Nat. Prod. 54, 1298-1302.

Spinas, G. A. (1999). The Dual Role of Nitric Oxide in Islet beta-Cells. News. Physiol. Sci. 14, 49-54. 